\title{
An Explanation for the Violation of Lepton Universality in Beauty-Quark Decays: The Binary Isotope Mixture of Beauty-Quarks
}

\author{
Ding-Yu Chung \\ Utica, Michigan, USA \\ Email: dy_chung@yahoo.com
}

How to cite this paper: Chung, D.-Y (2021) An Explanation for the Violation of Lepton Universality in Beauty-Quark Decays: The Binary Isotope Mixture of Beauty-Quarks. Journal of Modern Physics, 12, 971-982.

https://doi.org/10.4236/jmp.2021.127059

Received: April 1, 2021

Accepted: May 22, 2021

Published: May 25, 2021

Copyright $\odot 2021$ by author(s) and Scientific Research Publishing Inc. This work is licensed under the Creative Commons Attribution International License (CC BY 4.0).

http://creativecommons.org/licenses/by/4.0/

\begin{abstract}
This paper purposes an explanation for the recent evidence for the violation of lepton universality in beauty-quark decays at CERN's Large Hadron Collider. A beauty meson $\left(\mathrm{B}^{+}\right)$transforms into a strange meson $\left(\mathrm{K}^{+}\right)$with the emission of either electron-positron $\left(\mathrm{e}^{+} \mathrm{e}^{-}\right)$or muon-antimuon $\left(\mu^{+} \mu^{-}\right)$. The ratio $\left(R_{\mathrm{K}}\right)$ of branching fractions for $\mathrm{B}^{+} \rightarrow \mathrm{K}^{+} \mu^{+} \mu$ and $\mathrm{B}^{+} \rightarrow \mathrm{K}^{+} \mathrm{e}^{+} \mathrm{e}^{-}$decays is measured to be $R_{\mathrm{K}}=0.846$ instead of 1 in the violation of lepton universality in the Standard Model. This paper proposes that the violation is derived from the binary isotope mixture of two beauty-quarks, $b_{7}\left(4979 \mathrm{MeV}\right.$ mass) and $b_{8}$ (143,258 MeV mass) whose masses are calculated from the periodic table of elementary particles. $b_{7}$ is the observable $B$, while $b_{8}$ is the hidden $B$ to preserve the generation number symmetry between the three lepton family generations and the three quark family generations in the Standard Model. The preservation of the generation number symmetry forbids $b_{8}$ to decay into $\mathrm{K}^{+} \mu^{+} \mu^{\top}$. In the transition state involving the virtual particles $\left(\gamma, \mathrm{W} \pm\right.$ and $\left.\mathrm{Z}^{\circ}\right)$ before the decay, $b_{7}$ and $b_{8}$ emerge to form the binary isotope mixture from $B$. The rates of emergence as the rates of diffuse in Graham's law of diffusion are proportional to inverse square root of mass. The rate ratio between $b_{8} / b_{7}$ is $(4979 / 143,258)^{1 / 2}=0.1864$. Since $\mathrm{b}_{7}$ decays into $\mathrm{K}^{+}, \mathrm{e}^{+} \mathrm{e}^{-}$, and $\mu^{+} \mu^{\mu}$, while $\mathrm{b}_{8}$ decays into $\mathrm{K}^{+}, \mathrm{e}^{+} \mathrm{e}^{-}$, and forbidden $\mu^{+} \mu^{-}$, the calculated ratio $\left(R_{\mathrm{K}}\right)$ of branching fractions for $\mathrm{B}^{+} \rightarrow \mathrm{K}^{+} \mu^{+} \mu^{-}$and $\mathrm{B}^{+} \rightarrow \mathrm{K}^{+} \mathrm{e}^{+} \mathrm{e}^{-}$is $0.5 /(0.1864 \times 0.5+0.5)=0.843$ in excellent agreement with the observed 0.846 . The agreement between the calculated $R_{\mathrm{K}}$ and the observed $R_{\mathrm{K}}$ confirms the validity of the periodic table of elementary particles which provides the answers for the dominance of matter over antimatter, dark-matter, and the mass hierarchy of elementary particles.
\end{abstract}

\section{Keywords}

Beauty-Quark Decays, Violation of Lepton Universality, Periodic Table of 
Elementary Particles, Binary Isotope Mixture of Beauty-Quarks, Ratio of

Branching Fractions, Dark Matter, Dominance of Matter over Antimatter

\section{Introduction}

For about sixty years, the Standard Model (SM) of particle physics has provided the model for various properties and interactions of fundamental particles, and has been confirmed by numerous experiments. In lepton universality of the SM, the different charged leptons, the electron, muon and tau, have identical electroweak interaction strengths. Lepton universality has been confirmed in a wide range of particle decays. A recent measurement of beauty-quark decays based on proton-proton collision data collected with the LHCb detector at CERN's Large Hadron Collider shows the evidence for the breaking of lepton universality in beauty-quark decays [1]. A beauty meson $\left(\mathrm{B}^{+}\right)$transforms into a strange meson $\left(\mathrm{K}^{+}\right)$with the emission of leptons $\left(\ell^{+} \ell^{-}\right)$which is either electron-positron $\left(\mathrm{e}^{+} \mathrm{e}^{-}\right)$ or muon-antimuon $\left(\mu^{+} \mu^{-}\right)$. Lepton universality of the SM predicts that the ratio $\left(R_{\mathrm{K}}\right)$ of branching fractions for $\mathrm{B}^{+} \rightarrow \mathrm{K}^{+} \mu^{+} \mu^{-}$and $\mathrm{B}^{+} \rightarrow \mathrm{K}^{+} \mathrm{e}^{+} \mathrm{e}^{-}$decays is 1 . The observed $R_{\mathrm{K}}$ is 0.846 instead of 1 in the violation of lepton universality in the SM. The measurement has a significance of 3.1 standard deviations.

If confirmed by future measurements, this violation of lepton universality would imply new physics beyond the Standard Model. Since the SM is unable to explain cosmological observations of the dominance of matter over antimatter, dark-matter, or the mass hierarchy of elementary particles, the new physics derived from the violation of lepton universality can explain the SM's shortcomings. In the previous papers [2]-[8], the SM's shortcoming to account for the mass hierarchy of elementary particles is solved by the periodic table of elementary particles where the masses of all leptons, quarks, gauge bosons, the Higgs boson, and hadrons can be calculated precisely, and the calculated masses are in excellent agreement with the observed masses. As shown in this paper, the new physics from the violation of lepton universality actually confirms the validity of the periodic table of elementary particles.

This paper proposes that the violation is derived from the binary isotope mixture of two beauty-quarks, $b_{7}$ (4979 MeV mass) and $b_{8}(143,258 \mathrm{MeV}$ mass) whose masses are calculated from the periodic table of elementary particles [7]. $b_{7}$ is the observable $B$, while $b_{8}$ is the hidden $B$ to preserve the generation number symmetry between the three lepton family generations and the three quark family generations in the SM. The preservation of the generation number symmetry forbids $\mathrm{b}_{8}$ to decay into $\mathrm{K}^{+} \mu^{+} \mu$. In the transition state involving the virtual particles $\left(\gamma, \mathrm{W} \pm\right.$ and $\left.\mathrm{Z}^{\circ}\right)$ before the decay, $\mathrm{b}_{7}$ and $\mathrm{b}_{8}$ emerge to form the binary isotope mixture from $B$. The rates of emergence as the rates of diffuse in Graham's law of diffusion are proportional to inverse square root of mass. The rate ratio between $b_{8} / b_{7}$ is $(4979 / 143,258)^{1 / 2}=0.1864$. Since $b_{7}$ decays into $K^{+}, e^{+} e^{-}$, 
and $\mu^{+} \mu^{-}$, while $\mathrm{b}_{8}$ decays into $\mathrm{K}^{+}, \mathrm{e}^{+} \mathrm{e}^{-}$, and forbidden $\mu^{+} \mu^{-}$, the calculated ratio $\left(\mathrm{R}_{\mathrm{K}}\right)$ of branching fractions for $\mathrm{B}^{+} \rightarrow \mathrm{K}^{+} \mu^{+} \mu^{-}$and $\mathrm{B}^{+} \rightarrow \mathrm{K}^{+} \mathrm{e}^{+} \mathrm{e}^{-}$is $0.5 /(0.1864 \times 0.5+$ $0.5)=0.843$ in excellent agreement with the observed 0.846 . The agreement between the calculated $R_{\mathrm{K}}$ and the observed $R_{\mathrm{K}}$ confirms the validity of the periodic table of elementary particles.

Section 2 describes the periodic table of elementary particles and the calculation of the masses of leptons and quarks including the masses of $b_{7}$ and $b_{8}$. Section 3 describes the beauty-quark decay and the calculation of $R_{K}$ for the branching fractions of $\mathrm{B}^{+} \rightarrow \mathrm{K}^{+} \mu^{+} \mu^{-}$and $\mathrm{B}^{+} \rightarrow \mathrm{K}^{+} \mathrm{e}^{+} \mathrm{e}^{-}$. Section 4 explains dark matter and the dominance of matter over antimatter.

\section{The Periodic Table of Elementary Particles}

The periodic table of elementary particles for baryonic matter and dark matter [2]-[8] is based on the seven principal mass dimensions (d's) for stable baryonic matter leptons (electron and neutrinos), gauge bosons (all forces), gravity, and dark matter (five sterile dark matter neutrinos) and the seven auxiliary mass dimensions (a's) for unstable leptons (muon and tau) and quarks (d, u, s, c, b, and t) as in Figure 1 and Table 1.

Stable Baryonic Matter Leptons

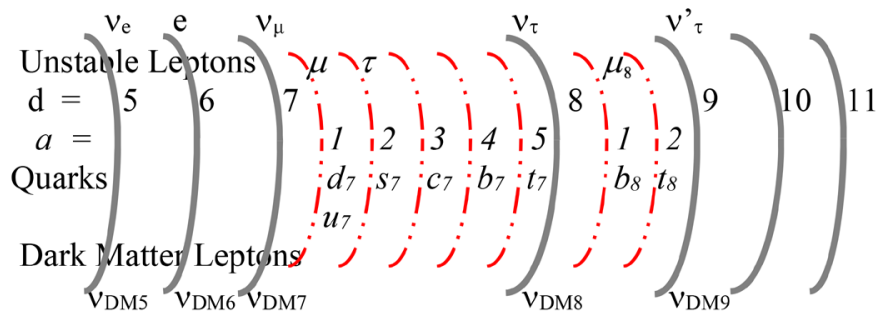

Figure 1. Leptons and quarks in the seven principal mass dimensional orbitals (solid lines) denoted by the principal mass dimensional orbital number $\mathrm{d}$ and the seven auxiliary dimensional mass orbitals (dash-dotted lines) denoted by the auxiliary mass dimensional orbital number $a$.

Table 1. The periodic table of elementary particles for baryonic matter and dark matter.

\begin{tabular}{|c|c|c|c|c|c|c|c|c|c|c|}
\hline & $a=0$ & $a=0$ & 1 & 2 & 1 & 2 & 3 & 4 & 5 & $a=0$ \\
\hline $\mathrm{d}$ & $\begin{array}{l}\text { Stable Baryonic } \\
\underline{\text { Matter Leptons }}\end{array}$ & $\frac{\text { Dark Matter }}{\underline{\text { Leptons }}}$ & $\begin{array}{l}\text { Unstable } \\
\underline{\text { Leptons }}\end{array}$ & & & \multicolumn{3}{|c|}{ Quarks } & & $\underline{\text { Bosons }}$ \\
\hline 5 & $v_{\mathrm{e}}$ & $\nu_{\mathrm{DM} 5}$ & & & & & & & & $\mathrm{~B}_{5}=\mathrm{A}$ electromagnetism \\
\hline 6 & $\mathrm{e}$ & $v_{\mathrm{DM} 6}$ & & & & & & & & $\mathrm{~B}_{6}=\mathrm{g}^{*}$ strong (basic gluon for quarks) \\
\hline 7 & $v_{\mu}$ & $\nu_{\mathrm{DM} 7}$ & $\mu_{7}$ & $\tau_{7}$ & $\mathrm{~d}_{7} / \mathrm{u}_{7}$ & $\mathrm{~S}_{7}$ & $\mathrm{c}_{7}$ & $\mathrm{~b}_{7}$ & $\mathrm{t}_{7}$ & $\mathrm{~B}_{7}=\mathrm{Z}_{\mathrm{L}}^{0}$ left-handed BM weak \\
\hline 8 & $\nu_{\tau}$ & WDM8 & $\mu_{8}$ (absent) & & $\mathrm{b}_{8}$ (absent) & $\mathrm{t}_{8}$ & & & & $\mathrm{~B}_{8}=\mathrm{Z}_{\mathrm{R}}^{0}$ right-handed DM weak \\
\hline 9 & $v_{\tau}\left(\right.$ high-mass $\left.v_{\tau}\right)$ & $\nu_{\mathrm{DM} 9}$ & & & & & & & & $\mathrm{~B}_{9}=$ dark matter repulsive force \\
\hline 10 & & & & & & & & & & $\mathrm{~B}_{10}=$ particle-antiparticle asymmetry \\
\hline 11 & Gravitino & & & & & & & & & $\mathrm{B}_{11}=$ gravity \\
\hline
\end{tabular}

$\mathrm{d}=$ principal mass dimension number, $\mathrm{a}=$ auxiliary mass dimension number, $\mathrm{DM}=$ dark matter, $\mathrm{BM}=$ baryonic matter. 
The periodic table of elementary particles provides the answers for the dominance of matter over antimatter [8], dark-matter [5], and the mass hierarchy of elementary particles [2]-[8]. The masses of all leptons, quarks, gauge bosons, the Higgs boson, gravity, and dark matter can be calculated by the periodic table of elementary particles [2]-[8]. Since this paper deals with mostly beauty quark, only the masses of leptons and quarks are calculated.

The mass of mass dimensional fermion and the mass of mass dimensional boson are related to each other with three simple formulas as the follows.

$$
\begin{gathered}
M_{\mathrm{d}, \mathrm{B}}=M_{\mathrm{d}, \mathrm{F}} / \alpha_{\mathrm{d}} \\
M_{\mathrm{d}+1, \mathrm{~F}}=M_{\mathrm{d}, \mathrm{B}} / \alpha_{\mathrm{d}+1} \\
M_{\mathrm{d}+1, \mathrm{~B}}=M_{\mathrm{d}, \mathrm{B}} / \alpha_{\mathrm{d}+1}^{2},
\end{gathered}
$$

where $\mathrm{d}$ is the mass dimension number, $\mathrm{F}$ is fermion, and $\mathrm{B}$ is boson. Each dimension has its own $\alpha_{\mathrm{d}}$, and all $\alpha_{\mathrm{d}}$ 's except $\alpha_{7}\left(\alpha_{w}\right)$ of the seventh dimension (weak interaction) are equal to $\alpha$, the fine structure constant of electromagnetism.

The lepton mass formula and the quark mass formula are derived from the incorporation of basic gluon $\left(\mathrm{g}^{*}=\mathrm{B}_{6}=M_{\mathrm{F} 6} / \alpha=M_{\mathrm{e}} / \alpha=70 \mathrm{MeV}\right.$ from Equation (1)) from Table 1 to electron. The incorporation of basic gluon as flux quanta follows the composite fermion theory for the FQHE (fractional quantum Hall effect) [9] [10]. In the composite fermion model for FQHE, the formation of composite fermion is through the attachment of an even number of magnetic flux quanta to electron, while the formation of composite boson is through the attachment of an odd number of magnetic flux quanta to electron. In the same way, the formation of composite fermion is through the attachment of an even number of basic gluons to electron, while the formation of composite boson is through the attachment of an odd number of basic gluons to electron. The formation of composite boson is equal to the formation of composite di-leptons, so the formation of composite lepton is through the attachment of one half of an odd number of basic gluons to electron. As a result, the muon $(\mu)$ mass formula is as follows.

$$
\begin{aligned}
M_{\mu_{7}} & =M e+3 M_{\mathrm{g}^{*}} / 2 \\
& =M e+3 M_{e} / 2 \alpha \\
& =105.5488 \mathrm{MeV}
\end{aligned}
$$

which is in excellent agreement with the observed $105.6584 \mathrm{MeV}$ [11] for the mass of muon. The masses of leptons follow the Barut lepton mass formula [12] as follows.

$$
M_{\text {lepton }}=M_{e}+\frac{3 M_{e}}{2 \alpha} \sum_{a=0}^{n} a^{4},
$$

where $a=0,1$, and 2 are for e, $\mu_{7}$, and $\tau_{7}$, respectively. The calculated mass of $\tau_{7}$ is $1786.2 \mathrm{MeV}$ in good agreement with the observed mass as $1776.82 \mathrm{MeV}$. According to Barut, the second term, $\sum_{a=0}^{n} a^{4}$ of the mass formula is for the 
Bohr-Sommerfeld quantization for a charge-dipole interaction in a circular orbit. The more precise calculated mass of $\tau$ for the tau lepton mass formula is as follows.

$$
\begin{aligned}
M_{\tau} & =M e+\left(\frac{3 M_{e}}{2 \alpha}-M_{e}\right) \sum 2^{4} \\
& =M e+\left(17 \frac{3 M_{e}}{2 \alpha}-17 M_{e}\right), \\
& =1777.47 \mathrm{MeV}
\end{aligned}
$$

which is in excellent agreement with observed $1776.82 \mathrm{MeV}$, and means that during this dipole-interaction in a circular orbit for $\tau$, an electron with total mass of $17 M_{e}$ is lost. $17 M_{e}$ is shown as the observed $17 \mathrm{MeV}$ for $34 M_{e}$ in the light boson (17 eē) [13] [14].

Quark has fractional charge $( \pm 1 / 3$ or $\pm 2 / 3)$, 3-color gluons (red, green, and blue) for $3 \mathrm{~g}^{\star}$, and both the principal mass dimensions and axillary mass dimensions, so similar to Equation (4), $\mathrm{d}$ and $\mathrm{u}$ in the principal mass dimension involves $\mathrm{e} / 3$ or $2 \mathrm{e} / 3$ and $3 \mathrm{~g}^{*}$ as follows.

principal mass dimensional oribital at $\mathrm{d}=6$

$$
\begin{aligned}
M_{\text {principal } q} & =\frac{1 \text { or } 2 M e}{3}+\frac{3\left(3 M_{\mathrm{g}^{*}}\right)}{2} \\
& =\frac{1 \text { or } 2 M e}{3}+\frac{3\left(3 M_{\mathrm{B} 6}\right)}{2} \\
& =\frac{1 \text { or } 2 M e}{3}+\frac{9 M e}{2 \alpha}
\end{aligned}
$$

For quarks in the auxiliary mass dimensions, 3 -color basic gluons $\left(3 g^{*}\right)$ become 3-color auxiliary basic gluons $\left(3 \mathrm{~g}_{\mathrm{a} 7}^{*}\right)$ at $\mathrm{d}=7$. From Equations (1) and (3), $\alpha_{W}=\alpha_{7}=\alpha$ of week interaction $=\left(M_{\mathrm{B} 6} / M_{\mathrm{B} 7}\right)^{1 / 2}=\left(M_{\mathrm{F} 6} / \alpha / M_{\mathrm{B} 7}\right)^{1 / 2}=\left(M_{e} / \alpha / M_{\mathrm{Z}}\right)^{1 / 2}=$ 0.02771. Based on Equation (2), auxiliary basic gluon is derived from muon as follows.

$$
M_{\mathrm{g}^{*} \mathrm{a}}=M_{\mu 7} \alpha_{w}
$$

Similar to Equation (4), the masses of quarks in the auxiliary mass dimension are as follows.

$$
\begin{aligned}
& \text { auxiliary mass dimensional orbital at } \mathrm{d}=7 \\
& M_{\text {auxiliary } q 7}=\frac{3\left(3 M_{\mathrm{g}_{\mathrm{a} 7} \mathrm{~T}}\right)}{2} \sum_{a=1}^{n} a^{4}=\frac{9 M_{\mu 7} \alpha_{w}}{2} \sum_{a=1}^{n} a^{4}
\end{aligned}
$$

The quark mass formula at $d=7$ is the combination of Equations (7) and (9) as follows.

$$
M_{q 7}=\frac{1 \text { or } 2 M e}{3}+\frac{9 M e}{2 \alpha}+\frac{9 M_{\mu 7} \alpha_{w}}{2} \sum_{a=1}^{n} a^{4}
$$

where $a=1,2,3,4$, and 5 for $\mathrm{u}_{7} / \mathrm{d}_{7}, \mathrm{~s}_{7}, \mathrm{c}_{7}, \mathrm{~b}_{7}$, and $\mathrm{t}_{7}$, respectively.

The quark mass at $a=5$ for the auxiliary mass dimension at $d=7$ is the maximum mass below the mass of $B_{7}$, so the next auxiliary mass dimension has 
to start from $B_{7}$. There are $b$ and $t$ at $d=8$, so it is necessary to have $\mu_{8}$ for the masses of $b$ and t. Like $\mu_{7}$ in Equation (4), the mass of $\mu_{8}$ is as follows.

$$
\begin{aligned}
M_{\mu_{8}^{0}} & =2 M e+3 M_{\mathrm{g}_{7}} / 2 \\
& =2 M e+3 M_{\mathrm{B} 7} / 2 \\
& =2 M e+3 M_{\mathrm{z}^{0}} / 2 \\
& =136.78 \mathrm{GeV}
\end{aligned}
$$

Since at $d=7$, there are 3-color basic gluons, at $d=8,3$-color basic gluons are not needed, and only one basic gluon $\left(\mathrm{g}^{*}\right)$ at $\mathrm{d}=7$ is used. Similar to Equations (7) and (9), the quark mass formulas for the principal and auxiliary mass dimensions are as follows.

$$
\begin{aligned}
& \text { principal mass dimensional orbital at } \mathrm{d}=7 \\
& M_{\text {principal quark }}=3 M_{\mathrm{g}_{7}} / 2=3 M_{\mathrm{B}_{7}} / 2=3 M_{\mathrm{Z}} / 2
\end{aligned}
$$

auxiliary mass dimensional orbital at $\mathrm{d}=8$

$$
M_{\text {auxiliary quark }}=\frac{3\left(M_{\mathrm{g}^{*}{ }_{\mathrm{a} 8}}\right)}{2} \sum_{a^{\prime}=1}^{n^{\prime}} a^{\prime 4}=\frac{3 \mu_{8}^{0} \alpha}{2} \sum_{a^{\prime}=1}^{n^{\prime}} a^{\prime 4}
$$

The quark mass formula at $d=8$ is the combination of Equations (10) and (11) as follows.

$$
M_{q 8}=\frac{3 M_{\mathrm{Z}}}{2}+\frac{3 M_{\mu_{8}^{0}} \alpha}{2} \sum_{a^{\prime}=1}^{n^{\prime}} a^{\prime 4}
$$

where $a^{\prime}=1$ and 2 for $b_{8}^{\prime}$ and $t_{8}^{\prime}$, respectively.

Combining Equations (10) and (14), the quark mass formula is as follows.

$$
M_{\text {quark }}=\frac{1 \text { or } 2 M e}{3}+\frac{9 M e}{2 \alpha}+\frac{9 M_{\mu_{7}} \alpha_{w}}{2} \sum_{a=1}^{n} a^{4}+\frac{3 M_{\mathrm{Z}}}{2}+\frac{3 M_{\mu_{8}^{0}} \alpha}{2} \sum_{a^{\prime}=1}^{n^{\prime}} a^{\prime 4}
$$

where $a=1,2,3,4$, and 5 for $\mathrm{d} / \mathrm{u}$. s, c, b, and t, respectively, and $\mathrm{a}^{\prime}=1$ and 2 for $\mathrm{b}$ and $\mathrm{t}$ respectively. The calculated masses ford, $\mathrm{u}, \mathrm{s}, \mathrm{c}, \mathrm{b}_{7}$, and $\mathrm{t}$ are $328.4 \mathrm{MeV}$, 328.6 MeV, 539.3 MeV, 1606.6 MeV, 4979.3 MeV, and 175.4 GeV, respectively. The calculated mass of $b_{8}\left(=b_{7}+b_{8}^{\prime}\right)$ is $143,258 \mathrm{MeV}$. In the SM, there are three generations of leptons. Extra-muon $\mu_{8}$ is outside of the three generations of leptons in the SM, so $\mu_{8}$ is absent as shown in Table 1 . As shown in Table 1 , to be symmetrical to the absent $\mu_{8}, b_{8}$ quark is also absent. In other words, $b_{8}$ quark is hidden. The calculated mass of top quark is $175.4 \mathrm{GeV}$ in good agreement with the observed $172.4 \mathrm{GeV}$ [11]. The periodic table of elementary particles calculates accurately the particle masses of all leptons, quarks, gauge bosons, hadrons, the Higgs boson, and the cosmic rays by using only five known constants: the number (seven) of the extra spatial dimensions in the observed four-dimensional space time from the eleven-dimensional membrane, the mass of electron, the masses of $\mathrm{Z}$ and $\mathrm{W}$ bosons, and the fine structure constant [5] [6] [7].

\section{Beauty-Quark Decays}

As described in the previous section, the mass of $b_{7}$ is $4974.6 \mathrm{MeV}$, while the 
mass of $b_{8}$ is $143,258 \mathrm{MeV}$. In the SM, there are three generations of leptons. Extra-muon $\mu_{8}$ is outside of the three generations of leptons in the $S M$, so $\mu_{8}$ is absent as shown in Table 1 . To be symmetrical to the absent $\mu_{8}, b_{8}$ quark is also absent. In other words, $b_{8}$ quark is hidden. As a result, the observable $b_{7}$ and the hidden $b_{8}$ form the binary isotope mixture.

A beauty meson $\left(\mathrm{B}^{+}\right)$transforms into a strange meson $\left(\mathrm{K}^{+}\right)$with the emission of leptons $\left(\ell^{+} \ell^{-}\right)$which is either electron-positron $\left(\mathrm{e}^{+} \mathrm{e}^{-}\right)$or muon-antimuon $\left(\mu^{+} \mu^{-}\right)$. The decay process includes a transition state where the decay process is mediated by virtual particles that can have a physical mass larger than the mass difference between the initial- and final-state particles. In the SM, these virtual particles include the electroweak-force carriers, the $\gamma, \mathrm{W} \pm$ and $\mathrm{Z}^{\circ}$ bosons, and the top quark as Figure 2.

In the transition state before the decay, from $\mathrm{B}^{+}$, the binary isotope mixture of beauty quarks emerges along with the virtual particles $\left(\gamma, \mathrm{W} \pm\right.$ and $\left.\mathrm{Z}^{\circ}\right)$ that can have a physical mass larger than the mass difference between the initial- and final-state particles. In the transition state, the binary isotope mixture is virtual. The rates of emergence as the rates of diffuse in Graham's law of diffusion are proportional to inverse square root of mass.

$$
R_{\mathrm{b}}=\left(M_{\mathrm{b}_{7}} / M_{\mathrm{b}_{8}}\right)^{1 / 2}=(4979 / 143258)^{1 / 2}=0.1864
$$

The preservation of the generation number symmetry to have the absent $\mu_{8}$ forbids $\mathrm{b}_{8}$ to decay into $\mathrm{K}^{+} \mu^{+} \mu^{-}$, while $\mathrm{b}_{7}$ can decay into $\mathrm{K}^{+} \mu^{+} \mu^{-}$and $\mathrm{K}^{+} \mathrm{e}^{+} \mathrm{e}^{-}$. As a result, $\mathrm{b}_{7}$ decays into $\mathrm{K}^{+}, \mathrm{e}^{+} \mathrm{e}^{-}$, and $\mu^{+} \mu^{-}$, while $\mathrm{b}_{8}$ decays into $\mathrm{K}^{+}, \mathrm{e}^{+} \mathrm{e}^{-}$, and forbidden $\mu^{+} \mu^{-}$. (The forbidden $\mu^{+} \mu^{-}$simply turns into $\gamma$.) The calculated ratio $\left(R_{\mathrm{K}}\right)$ of branching fractions for $\mathrm{B}^{+} \rightarrow \mathrm{K}^{+} \mu^{+} \mu^{-}$and $\mathrm{B}^{+} \rightarrow \mathrm{K}^{+} \mathrm{e}^{+} \mathrm{e}^{-}$is as follows.

$$
R_{\mathrm{K}}=0.5 /\left(0.5 R_{\mathrm{b}}+0.5\right)=0.5 /(0.5 \times 0.1864+0.5)=0.843
$$

The calculated ratio $\left(R_{\mathrm{K}}\right)$ of branching fractions for $\mathrm{B}^{+} \rightarrow \mathrm{K}^{+} \mu^{+} \mu^{-}$and $\mathrm{B}^{+} \rightarrow$ $\mathrm{K}^{+} \mathrm{e}^{+} \mathrm{e}^{-}$is 0.843 in excellent agreement with the observed 0.846 . The agreement between the calculated $R_{\mathrm{K}}$ and the observed $R_{\mathrm{K}}$ confirms the validity of the periodic table of elementary particles.

On molecular level, the emergence of the binary isotope mixture of $b_{7}$ and the hidden $b_{8}$ from $\mathrm{B}^{+}$is similar to the diffusion of the binary isotope mixture of

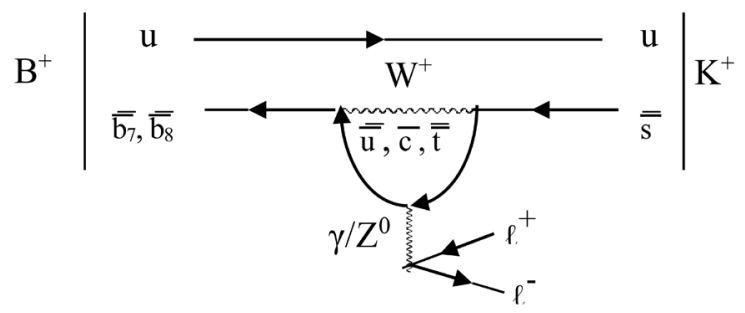

Figure 2. In the transition state before the decay, the binary isotope mixture of the two beauty quarks as the observable $\mathrm{b}_{\overline{7}}^{\overline{7}}\left(4979 \mathrm{MeV}\right.$ mass) and the hidden $\mathrm{b}_{\overline{8}}^{-}(143,258 \mathrm{MeV}$ mass) emergences from $\mathrm{B}^{+} . \mathrm{b}_{7}^{-}$decays into $\mathrm{K}^{+}, \mathrm{e}^{+} \mathrm{e}^{-}$, and $\mu^{+} \mu^{-}$, while $\mathrm{b}_{8}$ decays into $\mathrm{K}^{+}$and only $\mathrm{e}^{+} \mathrm{e}^{-}$. The virtual particles in the transition state include the electroweak-force carriers, the $\gamma, \mathrm{W} \pm$ and $\mathrm{Z}^{\circ}$ bosons, and the top quark. 
gaseous molecules. One of the well-known examples of the binary isotope mixture is the binary isotope mixture of ${ }^{3} \mathrm{He} /{ }^{4} \mathrm{He}$ from the degassing of the Earth's mantle through magmatism that results in the irreversible loss of helium to space, and high ${ }^{3} \mathrm{He} /{ }^{4} \mathrm{He}$ ratios observed in oceanic basalts have been considered the main evidence for a "primordial" undegassed deep mantle reservoir [15]. The initial ratio of the degassing rates from Graham's law between ${ }^{3} \mathrm{He} /{ }^{4} \mathrm{He}$ is as follows.

$$
R_{\mathrm{He}}=\left(M_{{ }^{4} \mathrm{He}} / M_{{ } \mathrm{He}}\right)^{1 / 2}=(4 / 3)^{1 / 2}=1.15
$$

As a result, the initial rate ratio of degassing is 1.15 which is confirmed by the observation. Another example of the binary isotope mixture is the neon isotope mixture of ${ }^{20} \mathrm{Ne} /{ }^{22} \mathrm{Ne}$ [16]. The isotopic diffusivity ratio for neon $\left({ }^{20} \mathrm{Ne} /{ }^{22} \mathrm{Ne}\right)$ in silicate glasses appears to equal the inverse square-root of the isotopic masses as 1.05 .

\section{Dark Matter and the Dominance of Matter over Antimatter}

It is speculated that since the SM is unable to explain cosmological observations of the dominance of matter over antimatter, the apparent dark-matter content of the Universe, or explain the patterns seen in the interaction strengths of the particles, the new physics derived from the violation of lepton universality can explain the SM's shortcomings [1]. In the Section 2, the periodic table of elementary particles explains the patterns seen in the interaction strengths of the particles for leptons and quarks. In this Section, dark matter and the dominance of matter over antimatter are explained by the periodic table of elementary particles.

The origins of dark matter and baryonic matter were explained in the references [5] [8] [17]. There are five types of dark matter and one type of baryonic matter with the mass ratio of 5:1 [8] [17]. In the periodic table of elementary particles, the five types of dark matter are the five sterile massive right-handed neutrinos, $v_{\mathrm{DM} 5}, v_{\mathrm{DM} 6}, v_{\mathrm{DM} 7}, v_{\mathrm{DM} 8}$, and $v_{\mathrm{DM} 9}[5]$. At $72.8 \%$ dark energy, the calculated values for baryonic matter and dark matter (with the 1:5 ratio) are $4.53 \%$ $(=(100-72.8) / 6)$ and $22.65 \%(=4.53 \times 5)$, respectively, in excellent agreement with observed $4.56 \%$ and $22.7 \%$, respectively [18].

The dominance of matter over antimatter can be explained by $B_{10}$ in the table for the principal mass dimensional bosons derived from the periodic table of elementary particles as in Table 2.

The lowest energy gauge boson $\left(B_{5}\right)$ at $d=5$ is the Coulomb field for electromagnetism. The second gauge lowest boson $\left(B_{6}\right)$ at $d=6$ is basic gluon $\left(g^{*}=70\right.$ $\mathrm{MeV} \approx$ one half of pion) is the strong force as the nuclear force in the pion theory [19] where pions mediate the strong interaction at long enough distances (longer than the nucleon radius) or low enough energies. $\mathrm{B}_{6}$ is denoted as basic gluon, $\mathrm{g}^{*}$. At short enough distances (shorter than the nucleon radius) or high enough energies, gluons emerge to confine fractional charge quarks. Fractional charge quarks are confined by gluons in QCD (quantum chromodynamics). No 
Table 2. The masses of the principal mass dimensional bosons (gauge bosons) $M_{\mathrm{d}, \mathrm{B}}=M_{\mathrm{d}, \mathrm{F}} / \alpha_{\mathrm{d}}$ and $M_{\mathrm{d}+1, \mathrm{~B}}=M_{\mathrm{d}, \mathrm{B}} / \alpha_{\mathrm{d}+1}^{2}$ from Equations (1) and (3).

\begin{tabular}{ccccc}
\hline $\mathrm{B}_{\mathrm{d}}$ & $M_{\mathrm{d}}$ & $\mathrm{GeV}$ (calculated) & Gauge boson & Interaction \\
\hline $\mathrm{B}_{5}$ & $M_{e} \alpha$ & $3.7 \times 10^{-6}$ & $\mathrm{~A}=$ photon & Electromagnetic \\
$\mathrm{B}_{6}$ & $M_{e} / \alpha$ & $7 \times 10^{-2}(70.02 \mathrm{MeV})$ & $\mathrm{g}^{*}=$ basic gluon & Strong \\
$\mathrm{B}_{7}$ & $M_{\mathrm{z}}=M_{\mathrm{B} 6} / \alpha_{\mathrm{w}}^{2}$ & 91.1876 (given) & $\mathrm{Z}_{\mathrm{L}}$ & weak (left) for baryonic matter \\
$\mathrm{B}_{8}$ & $M_{7} / \alpha^{2}=M_{\mathrm{z}} / \alpha^{2}$ & $1.71 \times 10^{6}$ & $\mathrm{Z}_{\mathrm{R}}$ & weak (right) for dark matter \\
$\mathrm{B}_{9}$ & $M_{8} / \alpha^{2}=M_{\mathrm{z}} / \alpha^{4}$ & $3.22 \times 10^{10}$ & & dark matter repulsive force \\
$\mathrm{B}_{10}$ & $M_{9} / \alpha^{2}=M_{\mathrm{z}} / \alpha^{6}$ & $6.04 \times 10^{14}$ & & particle-antiparticle asymmetry \\
$\mathrm{B}_{11}$ & $M_{10} / \alpha^{2}=M_{\mathrm{z}} / \alpha^{8}$ & $1.13 \times 10^{19}$ & $\mathrm{G}$ & gravity \\
\hline
\end{tabular}

isolated fractional charge quark is allowed, and only collective integer charge quark composites are allowed. In general, collective fractional charges are confined by the short-distance confinement force field where the sum of the collective fractional charges is integer [20]. As a result, fractional charges are confined and collective. The confinement force field includes gluons for collective fractional charge quarks in hadrons and the magnetic flux quanta for collective fractional charge quasiparticles in the fractional quantum Hall effect (FQHE) [21] [22] [23].

The third lowest boson $\left(B_{7}\right)$ at $d=7$ is $Z_{L}$ for the left-handed weak interaction among leptons and quarks. Massive weak bosons produce short-distance interaction. $B_{8}$ at $d=8$ is $Z_{R}$ for the right-handed weak interaction among dark matter neutrinos as dark matter neutrino oscillation. The symmetry between $Z_{R}$ and $Z_{\mathrm{L}}$ provides the neutrino oscillation for both baryonic matter neutrinos [24] and dark matter neutrinos.

$\mathrm{B}_{9}$ as the gauge boson represents dark matter repulsive force. The condensed baryonic gas at the critical surface density (derived from the acceleration constant $\mathrm{a}_{0}$ in MOND [25] [26]) induces the creation tensor for dark matter repulsive force to transform dark matter in the region into repulsive dark matter repulsing one another, corresponding to the Farnes' repulsive dark matter [8] [27]. Before the emergence of dark matter repulsive force, dark matter in the CMB was not repulsive.

$B_{10}$ at $d=10$ is for the gauge boson for particle-antiparticle asymmetry to provide the slight excess of particle in particle-antiparticle at the Big Bang, while $\mathrm{B}_{8}$ has particle-antiparticle symmetry. ( $B_{9}$ emerged long after the Big Bang.) As a result, the excess of particle is $\alpha^{4}\left(2.8 \times 10^{-9}\right)$ per particle-antiparticle (photon) for the ratio between $B_{8}$ and $B_{10}$. Since baryonic matter is $1 / 6$ of dark matter and baryonic matter [5] [8] [17], the baryonic matter excess is $4.7 \times 10^{-10}$ which is in a good agreement with $6 \times 10^{-10}$ for the ratio of the numbers between baryonic matter and photons in the Big Bang nucleosynthesis [28].

$B_{11}$ is for gravity. $F_{11}\left(8.275 \times 10^{16} \mathrm{GeV}\right)$ relates to spin $3 / 2$ gravitino, while $B_{11}$ 
$\left(1.134 \times 10^{19} \mathrm{GeV}\right)$ relates to spin 2 graviton. In supersymmetry, gravitino and graviton mediate the supersymmetry between fermion and boson in space dimension and gravitation. There are 11 space dimensions in the 11 space time dimensional membrane. As a result, the supersymmetry involves $11 \mathrm{~F}_{11}+\mathrm{B}_{11}$, which is equal to $1.225 \times 10^{19} \mathrm{GeV}$ in excellent agreement with the Planck mass $(1.221 \times$ $10^{19} \mathrm{GeV}$ ) derived from observed gravity as $(\hbar c / G)^{1 / 2}$ where $\mathrm{c}$ is the speed of light, $\mathrm{G}$ is the gravitational constant, and $\hbar$ is the reduced Planck constant.

\section{Summary}

In summary, this paper purposes an explanation for the recent evidence for the violation of lepton universality in beauty-quark decays at CERN's Large Hadron Collider. A beauty meson $\left(\mathrm{B}^{+}\right)$transforms into a strange meson $\left(\mathrm{K}^{+}\right)$with the emission of either electron-positron $\left(\mathrm{e}^{+} \mathrm{e}^{-}\right)$or muon-antimuon $\left(\mu^{+} \mu^{-}\right)$. The ratio $\left(R_{\mathrm{K}}\right)$ of branching fractions for $\mathrm{B}^{+} \rightarrow \mathrm{K}^{+} \mu^{+} \mu^{-}$and $\mathrm{B}^{+} \rightarrow \mathrm{K}^{+} \mathrm{e}^{+} \mathrm{e}^{-}$decays is measured to be $R_{\mathrm{K}}=0.846$ instead of 1 in the violation of lepton universality in the Standard Model. This paper proposes that the violation is derived from the binary isotope mixture of two beauty-quarks, $b_{7}\left(4979 \mathrm{MeV}\right.$ mass) and $b_{8}(143,258 \mathrm{MeV}$ mass) whose masses are calculated from the periodic table of elementary particles. $b_{7}$ is the observable $B$, while $b_{8}$ is the hidden $B$ to preserve the generation number symmetry between the three lepton family generations and the three quark family generations in the Standard Model. The preservation of the generation number symmetry forbids $\mathrm{b}_{8}$ to decay into $\mathrm{K}^{+} \mu^{+} \mu^{-}$. In the transition state involving the virtual particles $\left(\gamma, \mathrm{W} \pm\right.$ and $\left.\mathrm{Z}^{\circ}\right)$ before the decay, $\mathrm{b}_{7}$ and $\mathrm{b}_{8}$ emerge to form the binary isotope mixture from $B$. The rates of emergence as the rates of diffuse in Graham's law of diffusion are proportional to inverse square root of mass. The rate ratio between $b_{8} / b_{7}$ is $(4979 / 143,258)^{1 / 2}=0.1864$. Since $b_{7}$ decays into $\mathrm{K}^{+}, \mathrm{e}^{+} \mathrm{e}^{-}$, and $\mu^{+} \mu^{-}$, while $\mathrm{b}_{8}$ decays into $\mathrm{K}^{+}, \mathrm{e}^{+} \mathrm{e}^{-}$, and forbidden $\mu^{+} \mu^{-}$, the calculated ratio $\left(R_{\mathrm{K}}\right)$ of branching fractions for $\mathrm{B}^{+} \rightarrow \mathrm{K}^{+} \mu^{+} \mu^{-}$and $\mathrm{B}^{+} \rightarrow \mathrm{K}^{+} \mathrm{e}^{+} \mathrm{e}^{-}$is $0.5 /(0.1864 \times 0.5+0.5)=0.843$ in excellent agreement with the observed 0.846 . The agreement between the calculated $R_{\mathrm{K}}$ and the observed $\mathrm{R}_{\mathrm{K}}$ confirms the validity of the periodic table of elementary particles which provides the answers for the dominance of matter over antimatter, dark-matter, and the mass hierarchy of elementary particles.

\section{Conflicts of Interest}

The author declares no conflicts of interest regarding the publication of this paper.

\section{References}

[1] LHCb Collaboration (2021) Test of Lepton Universality in Beauty-Quark Decays. arXiv:2103.11769 [hep-ex]. https://arxiv.org/abs/2103.11769

[2] Chung, D. (1997) Speculations in Science and Technology, 20, 259-268. https://doi.org/10.1023/A:1026433207862 
[3] Chung, D. and Hefferlin, R. (2013) Journal of Modern Physics, 4, 21-26. https://doi.org/10.4236/jmp.2013.44A004

[4] Chung, D. (2016) Journal of Modern Physics, 7, 1591-1606. https://doi.org/10.4236/jmp.2016.712144

[5] Chung, D. (2018) Journal of Modern Physics, 9, 2308-2319. https://doi.org/10.4236/jmp.2018.913146

[6] Chung, D. (2018) Journal of Modern Physics, 9, 2638-2656. https://doi.org/10.4236/jmp.2018.914164

[7] Chung, D. (2019) Journal of Modern Physics, 10, 1310-1341. https://doi.org/10.4236/jmp.2019.1011087

[8] Chung, D. (2020) Journal of Modern Physics, 11, 1091-1122. https://doi.org/10.4236/jmp.2020.117069

[9] Kamilla, R., Wu, X. and Jain, J. (1996) Physics Review Letters, 76, 1332. https://doi.org/10.1103/PhysRevLett.76.1332

[10] Jain, J. (2007) Composite Fermions. Cambridge University Press, New York.

[11] Particle Data Group (2018) Physical Review D, 98, Article ID: 030001. https://doi.org/10.1103/PhysRevD.98.030001

[12] Barut, A. (1979) Physical Review Letters, 42, 1251. https://doi.org/10.1103/PhysRevLett.42.1251

[13] Krasznahorkay, A., et al. (2016) Physical Review Letters, 116, Article ID: 042501. https://doi.org/10.1103/PhysRevLett.116.042501

[14] Feng, J., et al. (2016) Physical Review Letters, 117, Article ID: 071803. https://doi.org/10.1103/PhysRevLett.117.071803

[15] Class, C. and Goldstein, S. (2005) Nature, 436, 1107-1112. https://doi.org/10.1038/nature03930

[16] Rama, S. and Hart, S. (1965) Science, 147, 737-738. https://doi.org/10.1126/science.147.3659.737

[17] Chung, D. and Krasnoholovets, V. (2013) Journal of Modern Physics, 4, 77-84. https://doi.org/10.4236/jmp.2013.47A1009

[18] Jarosik, N., et al. (2010) Seven-Year Wilson Microwave Anisotropy Probe (WMAP) Observations: Sky Maps, Systematic Errors, and Basic Results. http://lambda.gsfc.nasa.gov/product/map/dr4/pub papers/sevenyear/basic results/ wmap $7 \mathrm{yr}$ basic results.pdf

[19] Fujita, J. and Miyazawa, H. (1957) Progress of Theoretical Physics, 17, 360. https://doi.org/10.1143/PTP.17.360

[20] Chung, D. (2016) Journal of Modern Physics, 7, 1150-1159. https://doi.org/10.4236/jmp.2016.710104

[21] Tsui, D., Stormer, H. and Gossard, A. (1982) Physical Review Letters, 48, 1559 1562. https://doi.org/10.1103/PhysRevLett.48.1559

[22] Stormer, H. (1999) Reviews of Modern Physics, 71, 875-889. https://doi.org/10.1103/RevModPhys.71.875

[23] Laughlin, R. (1983) Physical Review Letters, 50, 1395-1398. https://doi.org/10.1103/PhysRevLett.50.1395

[24] Loureiro, A., et al. (2019) Physical Review Letters, 123, Article ID: 081301. https://doi.org/10.1103/PhysRevLett.123.081301

[25] Milgrom, M. (1989) Astronomy and Astrophysics, 211, 37-40. 
[26] Milgrom, M. (2009) Monthly Notices of the Royal Astronomical Society, 398, 1023 1026. https://doi.org/10.1111/j.1365-2966.2009.15255.x

[27] Farnes, J. (2018) Astronomy \& Astrophysics, 620, A92. https://doi.org/10.1051/0004-6361/201832898

[28] Steigman, G. (2007) Annual Review of Nuclear and Particle Science, 57, 463-491. https://doi.org/10.1146/annurev.nucl.56.080805.140437 